\title{
The Importance of the Climate Record in the Martian Polar Layered Deposits
}

\section{A White Paper submitted to the NASEM Planetary Science and Astrobiology Decadal Survey 2023-2032}

\begin{abstract}
Authors:
Patricio Becerra1 , Ali M. Bramson ${ }^{2,3}$, Adrian J. Brown ${ }^{4}$, Shane Byrne ${ }^{3}$, Andrea Coronato ${ }^{5}$, Serina Diniega ${ }^{6}$, Anna Grau Galofre ${ }^{7}$, Paul O. Hayne ${ }^{8}$, Briony Horgan², Christine S. Hvidberg' ${ }^{9}$, Margaret E. Landis ${ }^{8}$, Rachel W. Obbard $^{10}$, Alyssa Pascuzzo ${ }^{11}$, Jeffrey J. Plaut ${ }^{9}$, Ganna Portyankina ${ }^{8}$, Nathaniel E. Putzig ${ }^{12}$, Jorge Rabassa ${ }^{5}$, Isaac B. Smith ${ }^{12,13}$, Michael M. Sori ${ }^{2}$, Leslie Tamppari ${ }^{6}$, Nicolas Thomas ${ }^{1}$, Jennifer Whitten ${ }^{14}$

${ }^{1}$ Physics Institute, University of Bern (CH), ${ }^{2}$ Department of Earth, Atmospheric and Planetary Sciences, Purdue University, ${ }^{3}$ Lunar and Planetary Laboratory, University of Arizona, ${ }^{4}$ Plancius Research, ${ }^{5}$ Centro Austral de Investigaciones Científicas, CADIC-CONICET (AR), ${ }^{6}$ Jet Propulsion Laboratory, California Institute of Technology, ${ }^{7}$ School of Earth and Space Exploration, Arizona State University, ${ }^{\circ}$ Laboratory for Atmospheric and Space Physics, University of Colorado, Boulder, ${ }^{9}$ Niels Bohr Institute, University of Copenhagen (DK), ${ }^{10} \mathrm{SETI}$ Institute, ${ }^{11}$ Department of Earth, Environmental, and

Planetary Sciences, Brown University, ${ }^{12}$ Planetary Science Institute, ${ }^{13}$ Department of Earth and Space Science and Engineering, York University (CA), ${ }^{14}$ Department of Earth and Environmental Sciences, Tulane University
\end{abstract}

Primary contact information:

Patricio Becerra (patricio.becerra@space.unibe.ch, +41786976438)

Space Research and Planetary Science, Physics Institute, University of Bern $(\mathrm{CH})$

\section{Co-signatories:}

Timothy N. Titus, USGS

Kenneth E. Herkenhoff, USGS

Lauren A. Edgar, USGS

Sylvain Piqueux, NASA JPL/Caltech

Peter Buhler, NASA JPL/Caltech

Jonathan Bapst, NASA JPL/Caltech

John E. Moores, York U. (CA)

Shannon Hibbard, U. of Western Ontario (CA)

Chimira N. Andres, U. of Western Ontario (CA)

Aymeric Spiga, LMD, Sorbonne U. (FR)

Bernard Schmitt, IPAG, U. Grenoble Alpes (FR)

Matthew Chojnacki, Planetary Science Institute

Candice J. Hansen, Planetary Science Institute

Wendy Calvin, U. of Nevada, Reno

Antoine Pommerol, Physics Institute, U. of Bern $(\mathrm{CH})$

Clémence Herny, Physics Institute, U. of Bern $(\mathrm{CH})$
Camila Cesar, Physics Institute, U. of Bern $(\mathrm{CH})$

Jeremy Emmett, New Mexico State U.

Oded Aharonson, Weizmann Institute of Science (IL)

Eran Vos, Weizmann Institute of Science (IL)

Stefano Nerozzi, LPL, U. of Arizona

Eleni Ravanis, European Space Agency

Roberto Orosei, IRA-INAF, U. Bologna (IT)

Alice Lucchetti, OAPD-INAF, U. Padova (IT)

Maurizio Pajola, OAPD-INAF, U. Padova (IT)

Melinda Kahre, NASA Ames Research Center

Lauren McKeown, Natural History Museum (UK)

Daniel Lalich, Cornell U.

Ernst Hauber, DLR, Berlin (DE)

Zuriñe Yoldi, NBI, U. of Copenhagen (DK)

K.-Michael Aye, LASP, U. of Colorado Bouder

Frédéric Schmidt, U. Paris Saclay (FR)

Acknowledgements: A portion of the research was carried out at the Jet Propulsion Laboratory, California Institute of Technology, under a contract with the National Aeronautics and Space Administration (80NM0018D0004). 


\section{Summary}

Deciphering the climate record stored in the polar ice sheets of Mars is a high priority goal of the current Decadal Survey (2013-2023) and of the Mars Exploration Program Analysis Group (MEPAG). The goal of this white paper is to place focus on the importance of continued study of the stratigraphic record of climate in the Martian polar layered deposits (PLD) with follow-on missions that can fully test current theories. Our current knowledge of this record has enabled us to interpret the existence of astronomically-forced climatic variations on Mars, analogous to our own planet's Milankovitch cycles. This link has resulted in generic constraints and mature hypotheses on what the climate may have looked like in the recent past, which could be tested with future missions. Fully deciphering the polar record will require knowing the thickness of annual layers and absolute ages of specific strata, along with in situ measurements of the processes and materials that form a new layer. This will allow us to associate possible climate scenarios with specific accumulation or ablation periods, and thus accurately "read" the only other known detailed climate record on any planetary body besides Earth, in much the same way we read that of the Earth. Achieving this goal will have profound implications for our understanding of astronomically forced climates of planetary bodies, and it can help us infer whether Mars could have had habitable environments in the recent past.

\section{Background and Motivation}

The ice deposits of Mars are some of the most scientifically interesting features in the solar system due to their similarity to terrestrial ice, their accessibility for exploration, and their potential to contain a decipherable record of climate. The discovery of massive bodies of ice at the Martian poles by the Mariner 9 spacecraft led to an exponential development of the field of Mars Polar Science [Becerra et al. 2020a, Smith et al. 2020 WP]. Today we know that ice on Mars is exposed in the polar regions [Byrne, 2009], buried in the mid-latitudinal subsurface [Boynton et al. 2002; Bramson et al. 2020 WP], and possibly even present in equatorial locations [Wilson et al. 2017]. All of these ice deposits interact with climate on timescales ranging from months to tens of millions of years. The ices of Mars have thus been the topic of extensive research due to the wealth of climate information they contain and to their importance as records of past habitable environments. Furthermore, they could hold clues for how habitable environments could subsist today, and they have enormous potential for in-situ resource utilization (ISRU) by human exploration in the future [Bramson et al. 2020 WP]. The study of Martian ice is relevant to almost every topic of research in Mars science, from climatology - enabling the understanding of the fundamental mechanisms of climate change - to astrobiology - providing a record of the history of water, and by extension possible habitable environments, on Mars [Smith et al. 2018; 2020; 2020 WP].

Among the Martian ice reservoirs, the Polar Layered Deposits (PLD) (Fig.1) are the largest known bodies of water ice in the inner solar system outside of Earth [Byrne, 2009]. In many ways similar to Earth's polar ice sheets, these kilometers-thick domes of water ice and sediment (sand, silt, and dust) on the Martian poles have a stratified structure (Fig.1) long believed to be the product of shifts in climate that periodically change the amount of ice and sediment on the polar surfaces (Fig.1f) [Byrne, 2009]. These shifts are themselves a consequence of oscillations in Mars' astronomical parameters [Laskar et al. 2004], just as climate change on Earth depends on Milankovitch cycles [Hays et al. 1976]. Though significant progress has been made in the last decade connecting patterns in the PLD stratigraphy to Mars' astronomical oscillations, an accurate correlation of the polar layers to specific periods in Martian history - or even an accurate inventory of the layers present - has 
not been achieved. Establishing a reliable chronostratigraphic history of the PLD would mean understanding in detail how the Martian climate has evolved in geologically recent history. Furthermore, unlike Earth, Mars lacks additional complicating factors that contribute to climate change (oceans, a biosphere, human activity), so relating Martian climatic forcing to its stratified deposits will allow us to directly observe the geologic expression of the fundamental mechanisms of planetary climate change.

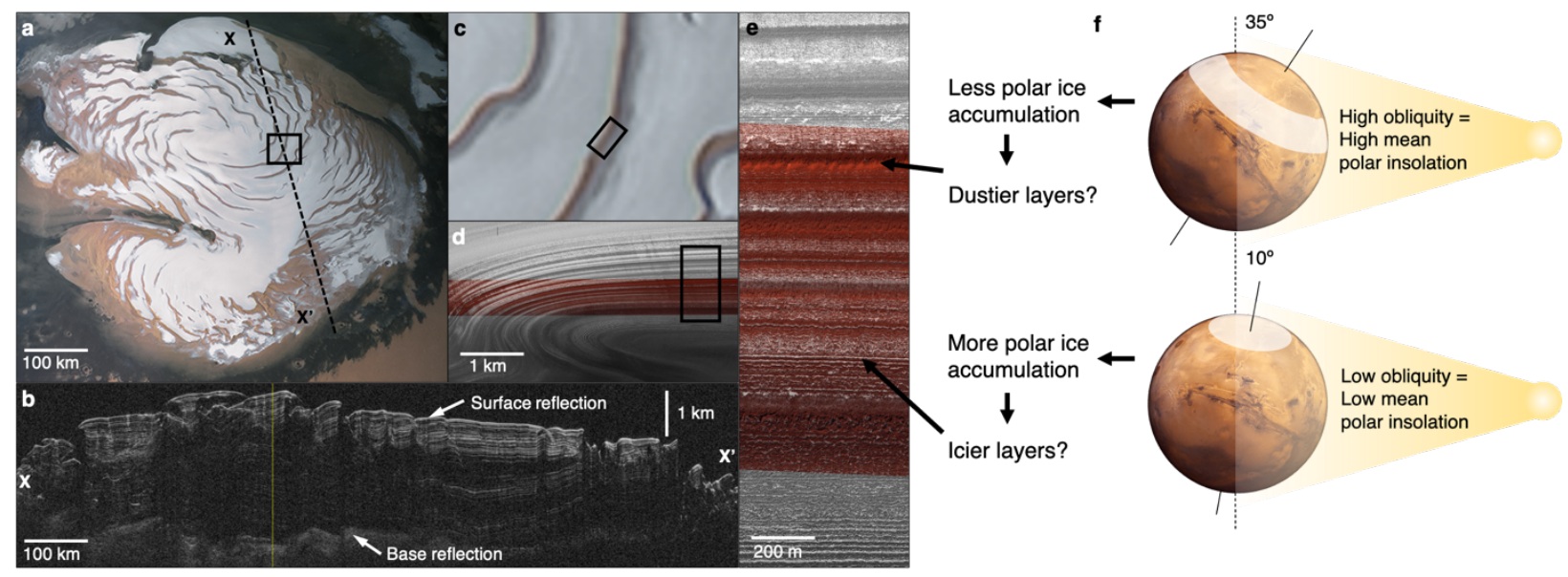

Figure 1. The north polar region of Mars. (a) View of Mars's NPLD by the High Resolution Stereo Camera (HRSC; ESA). Spiral troughs that expose internal layers are easily distinguishable. (b) Vertically exaggerated Shallow Radar (SHARAD) radar image of the NPLD. White bars denote scale. The dashed black line in (a) marks the surface-projected orbit track of the radar for this observation. (c) Close-up of a trough (box in (a)). (d) High Resolution Imaging Science Experiment (HiRISE) image of exposed layers in a trough (box in (c)). (e) Close-up of box in (d). Icy and dusty layers can be identified. (f) Schematic of obliquity-driven insolation changes for Mars. High polar insolation nominally means less ice accumulated at the poles in favor of the mid-latitudes, decreasing the ice/dust ratio of a polar ice layer, and vice versa.

Two methods of observation have generally been used to constrain the internal structure of the Martian PLD: direct imaging and subsurface radar. Direct imaging of the strata is possible due to the presence of extensive spiral troughs that dissect the ice sheets and expose the bedding (Fig.1) [Howard et al. 1982]. Radar sounders on the other hand, can "see through" the ice and observe the internal stratigraphy. The low permittivity of water ice allows the radar signal to penetrate, and the change in permittivity between icier and dustier layers results in partial reflections of the signal. In this way, radars observe the inside of the ice sheets as stacks of continuous, surface-parallel bands of reflections (Fig.1b) [Picardi et al. 2005; Phillips et al. 2008; Smith and Holt 2015].

Major steps toward understanding astronomical forcing of Martian climate have been taken in the last two decades. A combination of images, radar, gravity, and spectroscopic data as well as thermal and rheological arguments were used by various authors [Mellon et al. 1996; Nye et al. 2000; Picardi et al. 2005; Zuber et al. 2007] to determine that the bulk composition of the PLD is pure water ice with only small amounts of dust $(10 \%$ in the South [Picardi et al. 2005], and 5\% in the North [Grima et al. 2009]). Analysis of both images (Fig.1Ce) [Laskar et al. 2002; Milkovich and Head, 2005; Fishbaugh and Hvidberg, 2006; Limaye et al. 2012; Becerra et al. 2017,2019] and radar (Fig.1b) [Putzig et al. 2009; Smith et al. 2016; Whitten et al. 2017] have connected recurring patterns in the PLD layering to periodic oscillations of the Martian polar insolation due to orbital cycles. This resulted in estimates of net accumulation rates for the North PLD (NPLD), which in turn allowed the construction of models of its formation and accumulation based on the obliquity history over the last few million years [Levrard et al. 2007; Hvidberg et al. 2012] that can recreate several observed 
aspects of NPLD stratigraphy. Among numerous unconformities in the NPLD record observed in images [Tanaka et al. 2008], one particular unconformity detected in both images and radar was interpreted as a major erosional period followed by widespread accumulation [Smith et al. 2016], resembling a recent ice age and interglacial period predicted in models [Levrard et al., 2007]. This showed a general agreement between observations and models, resulting in mature hypotheses about the onset of NPLD accumulation and possible age estimates for stratigraphic columns [Hvidberg et al. 2012; Becerra et al. 2017].

However, there are key unknowns that prevent us from extending this knowledge to a detailed chronostratigraphic history of the PLD. First, although the bulk composition of the PLD is known, compositional differences between beds (which are most likely the reason a layered structure is visible) are not well constrained [Lalich et al. 2019]. Estimates of NPLD accumulation rates suggest that on average, annual layers are on the order of a mm thick, but we cannot observe these layers, as they are substantially below the resolution of current remote sensing. Notably, the relationship between the NPLD and South PLD (SPLD) is not understood. Although similar at first glance, they have striking differences that complicate the interpretation of a compound record of climate. Their surface ages are at least several orders of magnitude different (SPLD 10s of Myr [Herkenhoff \& Plaut, 2000; Koutnik et al. 2002]; NPLD as young as $1.5 \mathrm{kyr}$ [Banks et al. 2010; Landis et al. 2016]). The obliquity solutions used to model the formation of the NPLD are non-unique beyond $20 \mathrm{Ma}$, so the older age of the SPLD has only permitted estimates of its accumulation rate that are purely based on ratios of observed stratigraphic periodicities, and have a high degree of uncertainty [Becerra et al. 2019]. Furthermore, although the NPLD models (cited above) mostly agree that the NPLD began accumulating $\sim 4.2 \mathrm{Ma}$ (roughly coinciding with a drop in Mars' mean obliquity), the age of the SPLD cratering record, as well as recent modeling, suggest that polar ice could survive higher mean obliquities with only thin covers of insulating dust [Bramson et al. 2019]. In addition, the extent to which the much dustier SPLD built up a sublimation dust lag over single or multiple obliquity epochs, which could have major implications for our understanding of how buried ice survives on Mars, is not known. The nature and origin of sediments in the PLD is also not well understood. Visible/near-infrared spectral investigations have shown evidence for various salts as well as a significant mafic component, both of which are distinct from typical ferric dust [Horgan et al. 2009; Massé et al. 2012; Sinha et al. 2020]. This suggests that the PLD may preserve a record of recent impact, volcanic, and aqueous processes on Mars, as well as climate. Besides age and sediment content, the nature of the surface of each PLD is vastly different. Where the NPLD is covered in a residual cap of water ice that is likely the topmost layer of the NPLD record, the SPLD is partially coated by a cap of $\mathrm{CO}_{2}$ ice that interacts with climate on annual timescales [Byrne \& Ingersoll, 2003, Thomas et al. 2005]. In addition, beneath this $\mathrm{CO}_{2}$ coating are sequestered $\mathrm{CO}_{2}$ ice deposits (observed by SHARAD) that hold enough $\mathrm{CO}_{2}$ to double the mass of the current atmosphere [Phillips et al. 2011, Bierson et al. 2016]. These deposits likely represent a separate climate record, which may be connected to the residual cap, and whose nature we have only just begun to understand [Buhler et al. 2020].

In short, continuous exploration and research into the nature of the PLD climate record over the last 5 decades has resulted in major progress that has, at least in part, shaped our current comprehension of recent Martian geologic history. However, substantial research and exploration is still necessary to fully decipher the record, which would further define our view of Martian climate evolution. Additionally, a detailed understanding of the composition of the layers, including the possibility of liquid water layers [Orosei et al. 2018] could have profound 
implications for astrobiology and ISRU by crewed missions. We pose three overarching questions that we believe should be a focus of Mars exploration in the next decade, the answers to which will allow us to fully understand the geologically recent history of water and climate on a planet other than Earth, and help enable human exploration of Mars over the next half century:

1. What do the characteristics of Martian polar layered ice deposits reveal about their formation and evolution?

2. What is the absolute chronology of the observable climate records, and how do we interpret these records in terms of the Martian history of climate change and the possible existence of past habitable environments?

3. Why are the NPLD and SPLD so different in age, morphology, and interaction with current climate, and what does this tell us about Mars climate history?

\section{Critical knowledge gaps}

Goals documents put forth by the Mars Exploration Analysis Group (MEPAG), as well as the previous decadal survey, all list the Martian polar climate record as high priority science. For a detailed and current review of these goals and priorities from the viewpoint of the Mars Polar Science community, we reference the summary papers of the International Conferences on Mars Polar Science and Exploration (ICMPSE). These conferences have, since 1998, identified the most pressing gaps in knowledge regarding Mars ice research, and have driven the field forward through recommendations and published review papers [e.g. Smith et al. 2018]. The last conference was held in January of this year [Becerra et al. 2020a], and again identified the comprehensive interpretation of the polar climate record as a top priority. In order to address this and the overarching questions above, numerous subquestions and studies need to be tackled:

1. What do the characteristics of Martian polar ice deposits reveal about their formation and evolution?

(a) What are the energy and mass balances of the polar ice reservoirs, and how do they vary temporally and spatially?

(b) How does a layer form? What are the compositions of the layers, and how do they relate to the climate they formed in?

(c) How much sediment is present within or below the PLDs, what is its source, composition, and distribution, and what is its role and efficiency in the preservation of buried volatile reservoirs?

(d) What is the current distribution, morphology, and composition of the ice in the residual ice caps, and how do these characteristics relate to seasonal and annual processes?

(e) What is the thermal structure of the PLD and did it ever allow for fast enough ice flow to significantly affect the current state of the stratigraphic record?

(f) Can we confirm the presence of liquid water within the PLD at present or in the past [Orosei et al. 2018]?

2. What is the absolute chronology of the observable climate records, and how do we interpret these records in terms of the Martian history of climate change and the possible existence of past habitable environments?

(a) How does the observed astronomical climate forcing of the PLD translate into the resultant layer properties? 
(b) What is the absolute age of individual PLD layers, and are dateable impact ejecta or volcanic ash layers present in the PLD?

(c) What is the inventory of unconformities in the PLD record, and how do they relate to specific paleoclimatic scenarios? What is the volume/mass of the missing material?

3. Why are the NPLD and SPLD so different in age, morphology, and interaction with current climate, and what does this tell us about Mars climate history?

(a) Could the NPLD ice have existed before the drop in mean obliquity $\sim 4.2 \mathrm{Ma}$ ?

(b) Under what climatic conditions did the SPLD form? Given the SPLD's old surface age, and that recent climate is predicted to be unfavorable for south polar accumulation, under what conditions was it preserved?

(c) Is there an overlap in the climate records in the north and south poles?

3. How to address knowledge gaps in the next decade

In the last few years, a number of assessment reports and mission concepts have been put forward to determine the best approaches to answer many of the questions above. Reports by the MEPAG-led Ice and Climate Evolution Science Analysis Group [ICE-SAG, 2019; Diniega et al. 2020 WP], and the Next Orbiter Science Analysis Group [NEX-SAG, 2015, Campbell et al. 2020 WP] are especially relevant. A Keck Institute for Space Studies (KISS) workshop [Smith et al. 2020] inspired a NASA Discovery mission proposal (COMPASS) for a climate-and-ice focused orbiter mission that addresses some of the key knowledge gaps related to the polar record [Byrne et al. 2020]. Additionally, two Mars-climate-related proposals - MORIE [MORIE Final Report 2020] and MOSAIC [MOSAIC Final Report 2020] - were selected for funding through the 2019 Planetary Mission Concept Studies program. We list the most important strategies proposed that are relevant to the science questions above, and we relate these strategies to the knowledge gaps of section 2 in Table 1:

Table 1. Relationship between strategies (columns) and knowledge gaps (rows) in the study of the Martian polar climate record. All highlighted cells indicate that the strategy will help address the particular sub-question (see section 2). Cells highlighted in red indicate the most important and necessary strategy for that particular sub-question.

\begin{tabular}{|c|c|c|c|c|c|c|c|c|c|c|}
\hline \multicolumn{2}{|c|}{$\begin{array}{l}\text { Addressing the } \\
\text { knowledge gaps of } \\
\text { the Martian polar } \\
\text { climate record }\end{array}$} & $\begin{array}{l}\text { Ice core } \\
\text { extraction }\end{array}$ & $\begin{array}{l}\text { Outcrop } \\
\text { rovers }\end{array}$ & GPR & $\begin{array}{l}\text { Meteorology } \\
\text { station }\end{array}$ & LiDAR & $\begin{array}{l}\text { Higher } \\
\text { frequency } \\
\text { orbital } \\
\text { radar }\end{array}$ & $\begin{array}{l}\text { High-res } \\
\text { orbital } \\
\text { imaging and } \\
\text { spectroscopy }\end{array}$ & $\begin{array}{l}\text { Terrestrial } \\
\text { analogues }\end{array}$ & $\begin{array}{c}\text { Climate } \\
\text { simulations }\end{array}$ \\
\hline \multirow{6}{*}{$\begin{array}{l}\text { 1. Ice } \\
\text { characteristics }\end{array}$} & $1 \mathrm{a}$ & & & & & & & & & \\
\hline & $1 \mathrm{~b}$ & & & & & & & & & \\
\hline & $1 \mathrm{c}$ & & & & & & & & & \\
\hline & $1 \mathrm{~d}$ & & & & & & & & & \\
\hline & $1 \mathrm{e}$ & & & & & & & & & \\
\hline & $1 \mathrm{f}$ & & & & & & & & & \\
\hline \multirow{3}{*}{ 2. Chronology } & $2 a$ & & & & & & & & & \\
\hline & $2 b$ & & & & & & & & & \\
\hline & 2c & & & & & & & & & \\
\hline \multirow{3}{*}{$\begin{array}{l}\text { 3. NPLD vs. } \\
\text { SPLD }\end{array}$} & $3 a$ & & & & & & & & & \\
\hline & $3 b$ & & & & & & & & & \\
\hline & $3 c$ & & & & & & & & & \\
\hline
\end{tabular}

- Ice core extraction is the best way to obtain detailed climatic information from an ice sheet record on Earth. An analogous result could be achieved at Mars with a static lander carrying a drill that could deliver samples for analysis at the surface [Concept NF1 in ICESAG, 2019; Smith et al. 2020, Obbard et al. 2020]

- Micro computed tomography (microCT) is an example of an instrument that could be used for in-situ ice core analysis. It has been used to analyze the location and size/shape distribution of individual aeolian particles and layers in terrestrial ice sheets 
[Iverson et al. 2017]. A miniaturized microCT-based coring and analysis system could be used to characterize the porosity and distribution of sediment in the Martian PLD with higher resolution and to greater depths than is possible with any remote technique [Obbard et al. 2020; Edwards et al. 2020 WP].

- Ground Penetrating Radar (GPR, e.g. RIMFAX on Mars 2020) on a static lander would resolve the uppermost subsurface PLD beds over lateral distances up to a few $\mathrm{km}$. Alternatively (or additionally), a small rover could traverse the shallow slopes of troughs and study exposed layers in-situ, providing ground truth to orbital images and radar [Concept NF1 in ICE-SAG, 2019; Smith et al. 2020].

- A complete on-ground meteorology station would measure temperature, pressure and humidity, fully characterizing the atmospheric boundary layer and the rates of deposition and removal of ice and sediment in the current climate. In order to achieve this goal, landers that can survive the polar winter will be necessary to monitor surfaceatmosphere interactions throughout the year. These measurements are indispensable in order to understand the processes that lead to the formation of polar layers currently [Concepts NF1, NF2 in ICE-SAG, 2019; Smith et al. 2020].

- A multiple wavelength, active Near-IR LiDAR instrument has been proposed as a key orbital instrument that would measure the reflectance and polarization characteristics of the surface. Such an instrument would map the current polar deposition of $\mathrm{CO}_{2}$ and $\mathrm{H}_{2} \mathrm{O}$ ice during polar night contributing to our understanding of the current mass balance of the PLD [Brown et al. 2015, 2020 WP].

- A higher-frequency $(200-2000 \mathrm{MHz})$ orbital sounding radar has been almost universally accepted by the Mars ice community as a primary need within the next decade, as it is relevant to not just the polar ice record, but also to the subsurface mid-latitude ice record [Bramson et al. 2020 WP]. Although ongoing radar instruments (SHARAD and MARSIS) have proven crucial to the study of the polar record via their ability to observe the full stack of PLD strata from surface to base (Fig.1b), they fail to resolve the thinnest layers, and have not been correlated to the exposed layers seen in images of the PLD (Fig. 1e) [Christian et al. 2013; Becerra et al. 2020b]. A correlation between exposed beds and subsurface beds would result in the cap-wide characterization of the thinnest beds, an important step toward understanding bed formation, and thereby the construction of the climate record [NEX-SAG, 2015; Concept NF5 in ICE-SAG, 2019; Becerra et al. 2020b; Smith et al. 2020; MORIE Final Report 2020, MOSAIC Final Report 2020].

- Extending high-resolution orbital imaging, spectroscopy and stereotopography of Mars is critical to the questions above, and to all of Mars science. Many discoveries about the nature of the PLD beds and Martian climate forcing have been possible thanks to the degree of spatial and spectral resolution of instruments like MRO's HiRISE and CRISM [Concept NF5 in ICE-SAG, 2019; Smith et al. 2020; NEX-SAG, 2015, MORIE Final Report 2020, MOSAIC Final Report 2020].

- A planetary/Mars perspective on studies of terrestrial ice cores would help reveal the relations of radar response to layer composition, and to the morphology of exposed layers at the margins of ice sheets [Reeh, et al. 2002]. This is also relevant to mid-latitude ice and viscous flow features [Grau Galofre, et al. 2020 WP; Bramson et al. 2020 WP].

- Climate simulations with assimilation of new data (such as what would be acquired by some of the strategies above) will be essential to understand what types of environments generate specific types of layers, and thus answer questions related to the paleoclimatic conditions that formed the PLD. Current simulations with General 
Circulation Models (GCMs) [Levrard et al. 2007; Emmett et al. 2020] are limited by our knowledge of the current global climate (in particular winds and the vertical distribution of water vapor), but they can be updated to assimilate new data (as is being done with data from MAVEN [Stiepen et al. 2017] and ExoMars TGO [Young et al. 2019]) and thus better simulate a variety of climatic and paleoclimatic conditions. Climate simulations will singularly benefit from the new observations that we advocate for here.

\section{Concluding statement:}

To our current knowledge, Mars hosts the only detailed archive of climate change on any planet besides Earth. Aided by the relative simplicity of the Martian system, Martian polar ice comprises a global-scale laboratory for the study of the climatic evolution of terrestrial planets. Thus, deciphering the geological manifestation of Martian climate change as expressed in the polar record will impact studies in planetary science, geoscience, climate science, and, with the ongoing discovery of terrestrial exoplanets, even exoplanetary astronomy. This objective has consistently been listed as high-priority in analysis group reports and in the previous decadal survey. As we have demonstrated, new missions, both landed and orbital, will be necessary for the continued exploration of Martian Polar ice, and we strongly advocate for the prioritization and development of such missions. In the next decade, we have a unique opportunity to build upon prior achievements of Mars polar exploration, and read the climate record of another world for the first time.

\section{References}

\author{
Banks, M. E., et al. J Geophys Res Planets, 115, E08006 (2010) \\ Becerra, P., et al. Geophys Res Lett 44, 62-70 (2017). \\ Becerra, P. et al. Geophys Res Lett 121, 1-10 (2019). \\ Becerra, P., et al. Nat Astron 4, 566-568 (2020a) \\ Becerra, P. et al. $7^{\text {th }}$ ICMPSE, 6055 (2020b) \\ Bramson, A. M. et al. J Geophys Res Planets 124, 1020-1043 (2019). \\ Boynton, W.V. et al. Science 297, 81-85 (2002) \\ Brown, A.J. et al. J Quant Spect \& Rad Transfer, 153, 131-143 (2015) \\ Buhler et al. Nat Astron 4, 364-371 (2020) \\ Byrne, S., \& Ingersoll, A., Science, 299, 1051-1053 (2003) \\ Byrne, S. Ann. Rev. Earth Planet Sci 37, 535-560 (2009) \\ Byrne et al. $7^{\text {th }}$ ICMPSE, 6013 (2020) \\ Emmett et al. in press in Planet Space Sci, 105047 (2020) \\ Fishbaugh, K. E. \& Hvidberg, C. S. J Geophys Res 111, 26819-21 (2006). \\ Grima, C. et al. Geophys Res Lett 36, L03203 (2009). \\ Hays, J. D., et al. Science, 194(4270), 1121-1132 (1976) \\ Herkenhoff, K., and J. J. Plaut, Icarus, 144(2), 243-253 (2000) \\ Horgan, B. H., et al. (2009), J Geophys Res Planets, 114, E01005 (2009) \\ Howard et al. Icarus 50, 161-215 (1982) \\ Hvidberg, C. S. et al. Icarus 221, $405-419$ (2012) \\ Iverson, N. et al. Nat Sci Rep 7, 11457 (2017) \\ Koutnik, M., et al. J Geophys Res Planets, 107(E), 5100-1, (2002) \\ Lalich, D. E., et al. J Geophys Res Planets, 121(2), 1445-14 (2019) \\ Landis, et al. Geophys Res Lett, 43(7), 3060-3068 (2016) \\ Laskar, J., et al. Nature, 419(6905), 375-377, (2002) \\ Laskar, J., et al. Icarus, 170(2), 343-364, (2004) \\ Levrard, B., et al. J Geophys Res Planets, 112(E6), 5044-18, (2007) \\ Limaye, A. B. S., et al. J Geophys Res Planets 117, E06009 (2012). \\ Mellon et al. Icarus 124-1, 268-79 (1996) \\ Milkovich, S. M. \& Head, J. W. J Geophys Res Planets 110, E01005 \\ (2005) \\ Massé, M., et al. Earth and Planet Sci Lett 317, 44-55 (2012) \\ Nye, J. F., et al. Icarus, 144(2), 449-455 (2000) \\ Obbard, R.W. et al. 7th ICMPSE, $\underline{6078}(2020)$ \\ Orosei, R. et al. Science 117, eaar7268-8 (2018) \\ Picardi, G., et al. Planet Spac Sci, 52(1-3), 149-156, (2004) \\ Phillips et al., Science 320, 1182 (2008) \\ Phillips et al., Science 332, 838 (2011) \\ Putzig, N. E. et al. Icarus 204, 443-457 (2009) \\ Reeh, N. et al. Ann of Glaciology 35, 136-144 (2002)
}

Sinha et al. $7^{\text {th }}$ ICMPSE, $6077(2020)$

Smith, I. B. \& Holt, J. W. Nature 465, 450-453 (2010).

Smith, I. B., and J. W. Holt, J Geophys Res Planets, 120, E004720 (2015)

Smith, I. B. et al. Science 352(6289), 1075-1078 (2016)

Smith, I. B. et al. Icarus 308, 2-14 (2018).

Smith, I. B. et al. Planet Space Sci 184, 104841 (2020)

Stiepen, A. et al. J Geophys Res Space Physics, 122, $\underline{5782-5797}$

Tanaka, K. et al. Icarus 196, 318-358 (2008)

Thomas, P.C. et al. Icarus 174, $\underline{535-559}$ (2005)

Whitten, J. L., et al. Geophys Res Lett, 44(1), 8188-8195 (2017)

Wilson, J. T. et al. Icarus 299, 1-13 (2017).

Young, R.M.B. et al. Ninth International Conference on Mars, 6195 (2019)

Zuber M. et al., Science 317(5845), 1718-1719 (2007)

Reports:

MEPAG ICE-SAG Final Report (2019):

https://mepag.jpl.nasa.gov/reports/ICESAG Report FINAL.pdf MEPAG NEX-SAG Report (2015):

https://mepag.jpl.nasa.gov/reports/NEX-SAG draft v29 FINAL.pdf MORIE Final Report (2020): https://mepag.jpl.nasa.gov/meeting/202004/Day2/15 MORIE for MEPAG post.pdf

MOSAIC Final Report (2020): https://mepag.jpl.nasa.gov/meeting/202004/Day2/13 MOSAIC MEPAG 2020 short version minimal backup post.pdf

Other white papers submitted to this Decadal Survey:

Bramson et al. (2020) "Mid-Latitude Ice on Mars: A Science Target for Planetary Climate Histories and an Exploration Target for In Situ Resources"

Brown et al. (2020) "The case for a multi-channel polarization sensitive LIDAR for investigation of insolation-driven ices and atmospheres" Campbell et al. (2020) "Mars Next Orbiter Science Analysis Group" Diniega et al. (2020) "White Paper Summary of the Final Report from the Ice and Climate Evolution Science Analysis group (ICE-SAG)"

Edwards et al. (2020) "Emerging Capabilities for Mars Exploration"

Grau Galofre et al. (2020) "A Comparative View of Glacial and Periglacial Landforms on Earth and Mars"

Smith et al. (2020) "Solar-System-Wide Significance of Mars Polar Science" 